\title{
Effect of physical training periodization on physical activity level in adults with obesity
}

\author{
Efeito da periodização do treinamento físico no nível de atividade física em adultos \\ com obesidade
}

\begin{abstract}
AUTHOR'S
Guilherme Tadeu de Barcelos ${ }^{1}$ (D)

Giovani Firpo Del Duca ${ }^{1}$

Paulo Ricardo de Oliveira Medeiros ${ }^{1}$

Inácio Crochemore-Silva ${ }^{2}$ (D)

Aline Mendes Gerage ${ }^{1}$ (D)

1 Universidade Federal de Santa Catarina, Centro de Desportos, Departamento de Educação

Física, Grupo de Estudo e Pesquisa em Exercício

Físico e Doenças Crônicas Não Transmissíveis,

Florianópolis, Santa Catarina, Brazil.

2 Universidade Federal de Pelotas, Escola Superior de Educação Física, Grupo de Estudos e Pesquisas em Acelerometria, Pelotas, Rio Grande do Sul, Brazil.
\end{abstract}

\section{CORRESPONDING}

\section{Guilherme Tadeu de Barcelos}

guilherme_barcellos@hotmail.com

Universidade Federal de Santa Catarina,

Campus Universitário Reitor João David

Ferreira Lima, Grupo de Estudo e Pesquisa

em Exercício Físico e Doenças Crônicas Não

Transmissíveis. Florianópolis, Santa Catarina,

Brazil.

CEP: 88040-900.

DOI

$10.12820 /$ rbafs. $25 \mathrm{e} 0161$

\section{(cc) BY}

This work is licensed under a Creative Commons Attribution 4.0 International License.

\begin{abstract}
The study aimed to compare the effect of physical training with and without periodization on daily physical activity levels and on time spent in sedentary behavior in adults with obesity. In a randomized controlled trial, 69 obese adults of both sexes $\left(B M I \geq 30 \mathrm{~kg} / \mathrm{m}^{2}\right)$ were randomized to three groups: periodized group (PG), non-periodized group (NPG) and control group (CG). The periodized and non-periodized groups underwent 16 weeks of supervised and combined physical training (aerobic and resistance) in 60-minute sessions three times a week. Physical activity and sedentary behavior levels were measured by accelerometry (ActiGraph - GT3x). The time spent in sedentary behavior after the intervention increased $6.5 \%$ in the PG and $1.2 \%$ in the $C G$ and decreased $0.5 \%$ in the NPG. Changes in the levels of light physical activity were $-4.6 \%$ in the PG, $+0.5 \%$ in the NPG and $-2.3 \%$ in the $\mathrm{CG}$, while moderate to vigorous physical activity levels showed a reduction of $3.0 \%$ in the PG and increases of $0.5 \%$ in the NPG and $12.2 \%$ in the CG. It is worth mentioning, however, that no isolated time and group effects or time $\mathrm{x}$ group interaction were identified for any of the analyzed variables $(\mathrm{p}>0.05)$. A combined and supervised physical training program, with or without load progression, was not able to promote changes in the sedentary behavior and physical activity levels of obese adults, which shows that specific actions targeted at these behaviors are necessary.
\end{abstract}

Keywords: Obesity; Sedentary lifestyle; Physical exercise; Treatment.

\section{RESUMO}

O estudo teve por objetivo comparar o efeito do treinamento físico, com e sem periodização, nos níveis de atividade física diária e no tempo em comportamento sedentário de adultos com obesidade. Em um estudo randomizado controlado, 69 adultos de ambos os sexos com obesidade (IMC $\geq 30 \mathrm{~kg} / \mathrm{m}^{2}$ ) foram randomizados em três grupos: grupo periodizado (GP), grupo não periodizado (GNP) e grupo controle (GC). Os grupos periodizado e não periodizado foram submetidos a 16 semanas de treinamento físico combinado (aeróbio e resistido) e supervisionado, três vezes na semana, em sessões de 60 minutos. Os niveis de atividade física e comportamento sedentário foram mensurados por meio de acelerometria (ActiGraph - GT3x). O tempo despendido em comportamento sedentário após a intervenção aumentou $6,5 \%$ no GP e 1,2\% no GC e reduziu $0,5 \%$ no GNP. As mudanças nos níveis de atividade física leve foram de $-4,6 \%$ no GP, $+0,5 \%$ no GNP e $-2,3 \%$ no GC, ao passo que os niveis de atividade física moderada a vigorosa apresentaram reduçôes de 3,0\% no GP e aumentos de 0,5\% no GNP e 12,2\% no GC. Vale destacar, porém, que não foram identificados efeitos isolados do tempo e do grupo e nem interação tempo e grupo para nenhuma das variáveis analisadas ( $p$ > $0,05)$. Um programa de treinamento físico combinado e supervisionado, com ou sem progressão de carga, não foi capaz de promover mudanças no comportamento sedentário e nos niveis de atividade física de adultos com obesidade, sendo necessárias açôes especificas voltadas a estes comportamentos.

Palavras-chave: Obesidade; Sedentarismo; Exercício físico; Tratamento.

\section{Introduction}

Obesity affects approximately $13 \%$ of the world's adult population $^{1}$ and is characterized by excessive accumulation of adipose tissue due to multiple factors. This chronic noncommunicable disease is commonly associated with an imbalance between energy intake and expenditure, caused by high calorie consumption and low levels of physical activity $(\mathrm{PA})^{1,2}$. Lifestyle changes including the practice of physical exercises are funda- mental to its treatment ${ }^{3}$.

Combined physical training, characterized by the union of aerobic and muscle strength training in the same session, has been considered an effective way for treating obesity, due to the positive physiological and metabolic effects provided by the two training modalities 4 . One of its main benefits is the improvement in body composition, involving reductions in body fat percentage, increases in muscle strength and mass, and in 
$\mathrm{VO}_{2}$ peak $^{5}$. These results can be potentialized through an adequate manipulation of volume and intensity variables in a training program ${ }^{6}$. However, even when load progression is recommended during the training, the way in which this important variable should be manipulated during periodization in special population, as individuals with obesity, is not clear yet.

Additionally, many studies have highlighted the importance of increasing global PA levels and decreasing time spent in sedentary behavior (SB) due to the association of these factors with the incidence of chronic diseases and mortality ${ }^{7,8}$. Furthermore, research has reported on health damages resulting from prolonged $\mathrm{SB}$, and replacing the time spent in this behavior with an increase in PA levels is associated with improvements in different health-related parameters ${ }^{9,10}$. Therefore, it is important to explore different strategies that promote an increase in PA levels, especially in the population with obesity. However, ascertaining whether supervised programs of physical exercises - characterized by the organization and planning of the training variables ${ }^{11}$ - efficiently change the lifestyle of those who practice them has not been possible yet.

Considering that individuals with obesity remain in $\mathrm{SB}$ for longer periods compared to individuals with normal weight ${ }^{12}$, it is important to investigate if their engagement in a supervised physical training program would culminate in the adoption of healthier life habits regarding PA and SB. A previous study ${ }^{13}$ found a tendency of increase in energy expenditure deriving from $\mathrm{PA}$ in middle-aged men with overweight submitted to an aerobic training program. Based on this study, our hypothesis is that beginning a physical training program will influence behavior change positively, making individuals become more interested in adopting a healthier lifestyle, decreasing, for example, $\mathrm{SB}$ and increasing PA levels in a global way. Nevertheless, the literature indicates that the impact of physical training programs on the energy expenditure of the individuals on the energy expenditure of the individuals are highly variable, being influenced by sample characteristics like sex and age and also by components of the prescribed training, like duration of the session and of the intervention ${ }^{14}$. Manipulation or not of load progression has not been adequately investigated in this scenario. Therefore, the aim of this study was to compare the effect of combined training, with and without periodization, on daily PA levels and SB time of adults with obesity.

\section{Methods}

This study is a secondary analysis of data from a randomized controlled clinical trial called Projeto Movt (Mov+ Project), carried out between March and September of 2018 in the Sports Center of the Federal University of Santa Catarina. This larger study aimed to investigate the effects of two combined physical training models (aerobic and resistance) on different health parameters in adults with obesity. The components of health-related fitness were considered the primary outcomes in the larger study. In the present study, we analyzed the physical activity and sedentary behavior variables, considered secondary outcomes in the larger project. Details of the methods were also described in a recent study ${ }^{15}$.

The project was approved by the Ethics Committee of Research with Human Beings of Federal University of Santa Catarina, opinion number 2.448.674. The study was also registered in the platform Brazilian Clinical Trials Registry - ReBEC - under the code RBR-3c7rt3.

The sample calculation of the larger study ${ }^{15}$ was performed in the program Gpower 3.1 .7 , with the adoption of level of significance of 0.05 , statistical power of $80 \%$ and effect size of 0.18 , as well as a $1: 1: 1$ ratio for the groups (see the characterization of the groups below). The results of the calculation indicated a minimum of 26 participants in each group. Considering the possibility of sample losses during the intervention, 30 participants was the number stipulated to each group, totaling 90 individuals.

The intervention was disseminated through leaflets, posters and advertisements in the local media, and the participants were recruited in the vicinity of the place where the intervention would take place. After the dissemination, the individuals contacted the research team and scheduled interviews for an initial screening. The inclusion criteria were the following: all the participants must have a body mass index (BMI) corresponding to obesity degrees 1 (30 to $34.9 \mathrm{~kg} / \mathrm{m}^{2}$ ) and 2 (35 to 39.9 $\mathrm{kg} / \mathrm{m}^{2}$ ), must have been authorized by a physician to practice physical exercises, and could not have participated regularly in any physical exercise program in the last three months. In addition, the participants could not be smokers, excessive alcohol users ( $\geq 7$ doses per week for women and $\geq 14$ doses for men) nor users of medicines to control obesity. Furthermore, they could not have undergone bariatric surgery nor present limitations concerning muscles, bones or joints that could 
prevent the practice of physical exercises. All the participants signed a consent document in which they accepted the terms and their participation in the program.

The participants were submitted, initially, to baseline assessments, including sample characterization measures (sociodemographic data, height and body mass), assessment of SB and habitual PA (accelerometry measures), and other assessments that were part of the larger study ${ }^{15}$ but were not analyzed in the present one. After the assessments, the individuals were randomly assigned to three groups: training group with linear periodization (PG), non-periodized training group (NPG) and control group (CG). The randomization was performed by blocks through the Randomization software, in a 1:1:1 ratio, according to sex, age and BMI, based on a table of random numbers. It was conducted by researchers who were not directly involved in the recruitment of participants and did not have access to the baseline assessments.

The exercise groups were submitted to 16 weeks of combined physical training (aerobic and resistance exercises in the same session) performed in 60-minute group sessions, three times per week, in non-consecutive days. The first week was reserved for familiarization with the exercises and with the training model. Then, the training period was divided into three mesocycles of five weeks each. In the aerobic training, the walking and/or running modalities were used, while the resistance training was composed of weightlifting exercises with dynamic exercises in machines and free weights for upper and lower limbs.

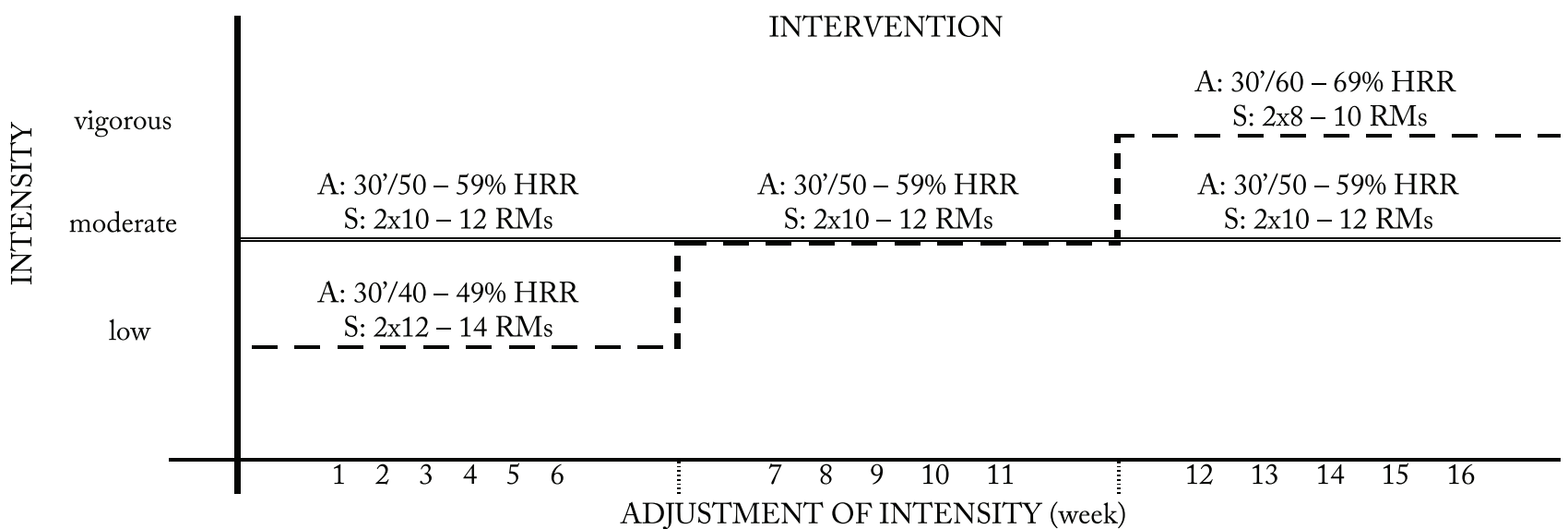

- - - : periodized group;

$\overline{-}$ : non-periodized group;

A: aerobic training;

S: strength training;

HRR: heart rate reserve;

$\mathrm{RMs}=$ repetitions maximum; $\mathrm{x}$ : series.

Figure 1 - Training periodization.
Intensity control in the aerobic training was provided by the percentages of the heart rate reserve (HRR), calculated by means of the resting heart rate and maximum heart rate, and obtained through an incremental test, as proposed by Jones \& Doust ${ }^{16}$. The intensity of the resistance training was controlled by repetition maximum zones.

The PG sessions were composed of five initial minutes of warm-up exercises, 50 minutes in which the participants practiced aerobic exercises (30 minutes) and resistance exercises (20 minutes), and five final minutes for relaxation, with stretching exercises. The training of this group was characterized by relative load progression during the 16 weeks of training: the first mesocycle was performed with low intensity, the second with moderate intensity, and the third with vigorous intensity. NPG followed the same organization of the PG training sessions, but the 16 weeks of training were composed by the same training intensity, without relative load progression. During the intervention, the intensity of the aerobic training was readjusted, with a re-assessment of the HRR and maintenance of the relative training intensity. In the resistance training, the absolute load was readjusted. As a way of controlling the participants' engagement in the training program, the number of sessions attended by each participant was registered and a percentage was calculated based on the total number of sessions. In the results, we indicated how many participants adhered to, at least, 50 and $70 \%$ of the training sessions.

$\mathrm{CG}$, in turn, was not submitted to any intervention

\section{INTERVENTION}

ADJUSTMENT OF INTENSITY (week) 
and was instructed to maintain their usual routine. After the intervention period, all the CG participants were invited to participate in the training carried out with PG and NPG.

For the purpose of sample characterization, sociodemographic data and anthropometric measures were collected before the beginning of the intervention. The sociodemographic variables sex and age (in years) were obtained by means of an online questionnaire answered in the Question Pro platform. Body mass (kg) was measured with an electronic scale (Welmy, model W300A), accurate to $100 \mathrm{~g}$, and height was measured with a stadiometer connected to the scale, accurate to $0.1 \mathrm{~cm}$. BMI was calculated from the height and body mass measures.

To assess the participants' PA levels, accelerometers (ActiGraph ${ }^{\circledR}$, model GT3X+) were fixed on the right side of the waist, above the iliac crest. Data were collected at the frequency of $100 \mathrm{~Hz}$ and analyzed in 60-second epochs.

All the participants were instructed to wear the device during seven consecutive days, removing it only to sleep, bathe and perform water activities. The participants included in the analysis were those who wore the device for no less than four days (at least one of the days must be on the weekend) during a minimum of 10 hours per day. The assessments were performed before the beginning of the training program (baseline) and after the end of the 16 weeks of training (post-intervention), in order to avoid evaluating the time spent in activities of the proposed training program. All the participants were instructed to maintain their usual routine of daily physical activities.

The data were extracted and analyzed in the software ActiLife. We used the cut-off points proposed by Freedson et al. ${ }^{17}$ for SB and by Sasaki et al. ${ }^{18}$ for light PA (LPA) and moderate to vigorous PA (MVPA) (primary outcome). These variables were treated in a continuous way, in minutes/day, being adjusted according to the daily period of time during which the accelerometer was worn and to the number of valid days. In addition, considering the cut-off points, the number of times per day and the total time (minutes/day) spent in SB bouts and in MVPA bouts were calculated. Bouts were defined as continuous periods spent in the same zone of intensity (SB or MVPA) with minimum duration of 10 minutes without interruption and tolerance of 2 minutes (spike tolerance ${ }^{19}$. We also evaluated the number (times/day) of SB breaks, defined as periods of at least two minutes of interruption of a certain behavior.

In the data analysis, the software Statistical Package for the Social Sciences (SPSS for Windows, Version 21.0) was used. In the descriptive statistics, the continuous variables were expressed in mean and standard deviation, while the categorical variables were presented in absolute and relative frequency. Data distribution was checked by the Shapiro-Wilk test. One-way Anova and the chi-square test were used to compare groups at baseline, regarding the continuous and categorical variables, respectively. For the intra- and inter-groups comparison of the $\mathrm{SB}$ and $\mathrm{PA}$ variables, we applied a two-way repeated measures ANOVA, considering the factors group (intervention vs. control) and time (baseline vs. post-intervention). The Bonferroni post hoc test was used to identify possible differences between variables, after the sphericity assumptions were confirmed by means of the Mauchly's test.

An intention-to-treat analysis was carried out, so that all the randomized participants with valid accelerometry data at baseline were included in data analysis. In the case of missing data in the post-intervention moment - individuals who gave up on the program or non-valid accelerometry data -, imputation was performed through the technique of replacing the missing value with the last observed value $\mathrm{e}^{20-22}$. A per protocol analysis was also carried out, including only the participants who completed the program and whose accelerometry data were validated.

\section{Results}

Figure 2 presents the study's flowchart. Initially, 515 people were interested in participating in the study. However, 432 were excluded because they did not meet the inclusion criteria. A total of 83 individuals met all the eligibility criteria, but 14 individuals were excluded because they decided not to participate or because they were not assessed in the pre-intervention period. Therefore, 69 participants were randomized to the groups PG $(n=23), N P G(n=23)$ and CG $(n=23)$. After the randomization and baseline assessments, nine participants with non-valid accelerometry data were identified and were not included in the final analysis of the present study. Concerning adherence to the training sessions, eight NPG participants and nine PG participants adhered to $>50 \%$ of the training sessions and, of these individuals, only three NPG participants and four PG participants adhered to $>70 \%$ of the training sessions. Regarding dropouts, 27 participants did not 
complete the program. There were no statistically significant differences for adherence nor for weekly attendance between the investigated groups (non-published data). The most cited reason by the dropouts was related to personal issues, unrelated to the training, over time $(n=18)$. When we compared the baseline characteristics of the participants who completed the program $(\mathrm{n}=33)$ with those of the individuals who gave up on the program $(n=27)$, we did not find significant differences related to sex $(p=0.852)$, time spent in SB $(\mathrm{p}=0.749)$ and level of MVPA $(\mathrm{p}=0.854)$. However, we found significant differences related to the age of the participants who completed the program (37.5 \pm 6.1) compared to the age of those who did not complete it $(32.0 \pm 7.7)(\mathrm{p}=0.003)$.

In the intention-to-treat analysis, 60 adults were included: men and women aged between 20 and 50 years. The largest part of the sample was composed of women (61.7\%) and the participants' mean age was 37.8 years, without difference between groups. BMI presented a mean value of $32 \mathrm{~kg} / \mathrm{m}^{2}$ and there was also no difference between groups (Table 1 ).

In relation to levels of LPA, MVPA and SB time, no significant differences were found between groups at baseline $(p=0.203 ; p=0.294 ; p=0.142$, respectively). The intra- and inter-groups analysis of the average daily time spent in SB, LPA and MVPA did not identify a statistically significant effect for time, group or group $\mathrm{x}$ time interaction. As for SB and MVPA bouts and SB breaks, no statistically significant intra- or inter-groups

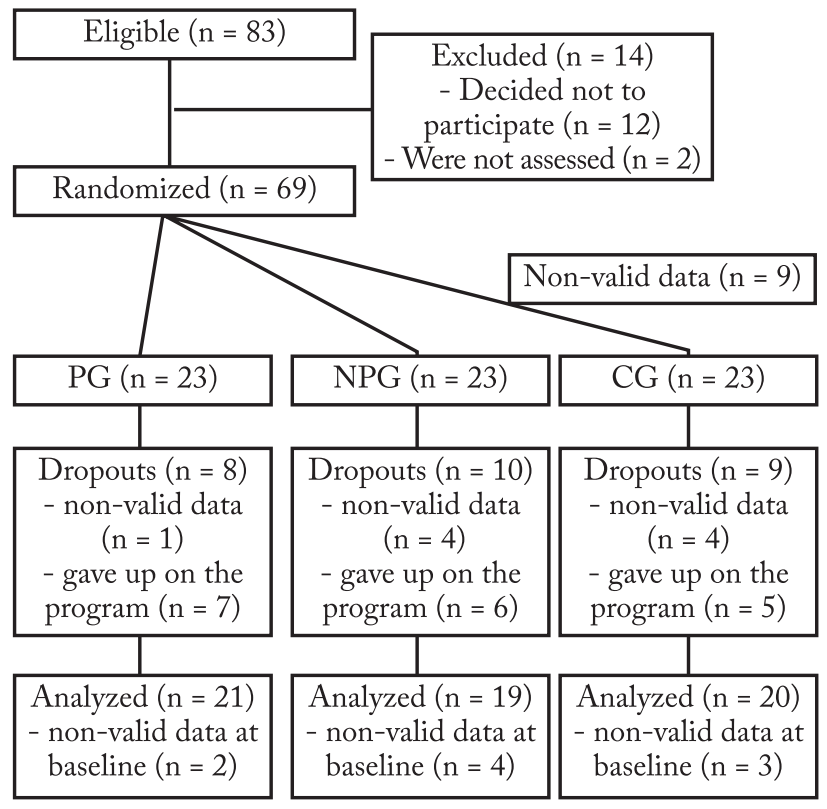

Figure 2 - Study's flowchart. differences were identified $(\mathrm{p}>0.05)$ - Table 2 .

Table 1 - Baseline comparison of sociodemographic and body composition characteristics of adults with obesity in the different groups $(n=60)$.

\begin{tabular}{lcccc}
\hline & $\begin{array}{c}\text { PG } \\
(\mathrm{n}=21)\end{array}$ & $\begin{array}{c}\text { NPG } \\
(\mathrm{n}=19)\end{array}$ & $\begin{array}{c}\text { CG } \\
(\mathrm{n}=20)\end{array}$ & p-value \\
\hline $\begin{array}{l}\text { Sex } \\
(\% \text { female })\end{array}$ & 57.1 & 63.2 & 65.0 & $0.863^{+}$ \\
$\begin{array}{l}\text { Age } \\
( \pm \text { sd, years })\end{array}$ & $36.14 \pm 7.50$ & $33.89 \pm 7.22$ & $34.85 \pm 7.59$ & $0.633^{++}$ \\
$\begin{array}{l}\text { Body mass } \\
\left(\bar{x}^{ \pm} \text {sd, kg }\right)\end{array}$ & $98.38 \pm 17.49$ & $98.34 \pm 14.43$ & $94.68 \pm 11.97$ & $0.667^{++}$ \\
$\begin{array}{l}\text { Height } \\
(\bar{x} \pm \text { sd, cm })\end{array}$ & $1.70 \pm 0.11$ & $1.69 \pm 0.10$ & $1.68 \pm 0.09$ & $0.892^{++}$ \\
$\begin{array}{l}\text { BMI } \\
\left(\bar{x}^{ \pm} \mathrm{sd}, \mathrm{kg} / \mathrm{m}^{2}\right)\end{array}$ & $33.81 \pm 3.24$ & $34.00 \pm 3.15$ & $33.25 \pm 2.41$ & $0.709^{++}$ \\
\hline
\end{tabular}

Note: $\mathrm{PG}=$ periodized group; $\mathrm{NPG}=$ non-periodized group; $\mathrm{CG}$ = control group; $\mathrm{sd}$ = standard deviation; $\mathrm{BMI}=$ body mass index; $\dagger=$ analysis by means of chi-square test; $\dagger \dagger=$ analysis by means of one-way Anova.

In the per protocol analysis, conducted only with the participants who started and completed the program, the results were similar to those found in the intention-to-treat analysis for all the analyzed variables (no intra- and inter-groups changes; $\mathrm{p}>0.05$ ).

When we analyzed PG and NPG jointly and compared the results to $\mathrm{CG}$, we did not identify isolated effects of group, time and group $\mathrm{x}$ time interaction $(\mathrm{p}$ $>0.05$ ) for any of the analyzed variables, neither in the intention-to-treat analysis nor in the per protocol analysis. Net-effect ( $\Delta$ control $-\Delta$ of PG and NPG) was $-5.8 \%, 0.3 \%$ and $32.8 \%$ for the variables SB, LPA and MVPA, respectively (per protocol analysis).

\section{Discussion}

The present study aimed to compare the effect of a 16week supervised program of combined physical training, with and without progression, on PA and SB levels of adults with obesity. The results showed that this kind of training, independently of progression, was not able to produce significant changes in this population's SB time and PA levels.

Studies that investigated the impact of supervised physical training programs on PA levels of individuals with obesity have provided inconclusive results ${ }^{14,23}$. Fedewa et al. ${ }^{14}$, in a review study, found that, in general, physical training does not produce significant changes in habitual PA levels, but the authors emphasized that the majority of the studies involved aerobic training and only a few investigated the effects of resistance and/or combined training. Therefore, changes in habit- 
Table 2 - Comparison of means of physical activity levels, sedentary behavior, bouts and breaks between groups $(n=60)$.

\begin{tabular}{|c|c|c|c|c|c|c|}
\hline & \multirow{2}{*}{$\mathrm{PG}(\mathrm{n}=21)$} & \multirow{2}{*}{ NPG $(\mathrm{n}=19)$} & \multirow{2}{*}{$C G(n=20)$} & time & group & $t^{*} g$ \\
\hline & & & & \multicolumn{3}{|c|}{$\mathrm{p}$-value } \\
\hline \multicolumn{7}{|c|}{$\mathrm{SB}(\min /$ day $)$} \\
\hline Pre & $389.67 \pm 109.37$ & $461.46 \pm 103.98$ & $417.76 \pm 125.02$ & \multirow{3}{*}{0.162} & \multirow{3}{*}{0.221} & \multirow{3}{*}{0.198} \\
\hline Post & $414.97 \pm 116.84$ & $458.92 \pm 90.76$ & $422.62 \pm 100.67$ & & & \\
\hline$\Delta \%$ & 6.5 & -0.5 & 1.2 & & & \\
\hline \multicolumn{7}{|c|}{ LPA (min/day) } \\
\hline Pre & $504.04 \pm 94.28$ & $448.08 \pm 94.36$ & $487.88 \pm 109.99$ & \multirow{3}{*}{0.122} & \multirow{3}{*}{0.313} & \multirow{3}{*}{0.327} \\
\hline Post & $480.73 \pm 104.19$ & $450.35 \pm 81.34$ & $476.37 \pm 85.09$ & & & \\
\hline$\Delta \%$ & -4.6 & 0.5 & -2.3 & & & \\
\hline \multicolumn{7}{|c|}{ MVPA (min/day) } \\
\hline Pre & $66.28 \pm 34.67$ & $50.46 \pm 39.12$ & $54.37 \pm 24.35$ & \multirow{3}{*}{0.522} & \multirow{3}{*}{0.328} & \multirow{3}{*}{0.358} \\
\hline Post & $64.29 \pm 31.71$ & $50.73 \pm 34.69$ & $61.00 \pm 27.78$ & & & \\
\hline$\Delta \%$ & -3.0 & 0.5 & 12.2 & & & \\
\hline \multicolumn{7}{|c|}{ Daily amount of SB bouts (times/day) } \\
\hline Pre & $14.75 \pm 4.49$ & $17.03 \pm 3.79$ & $15.03 \pm 5.39$ & \multirow[t]{2}{*}{0.410} & \multirow[t]{2}{*}{0.261} & \multirow[t]{2}{*}{0.729} \\
\hline Post & $14.59 \pm 4.12$ & $16.46 \pm 3.62$ & $15.03 \pm 4.37$ & & & \\
\hline \multicolumn{7}{|c|}{ Total SB time in bouts (min/day) } \\
\hline Pre & $303.44 \pm 122.33$ & $382.20 \pm 118.39$ & $330.74 \pm 139.60$ & \multirow[t]{2}{*}{0.993} & \multirow[t]{2}{*}{0.255} & \multirow[t]{2}{*}{0.161} \\
\hline Post & $324.00 \pm 128.13$ & $366.37 \pm 107.34$ & $326.20 \pm 113.64$ & & & \\
\hline \multicolumn{7}{|c|}{ Daily amount of MVPA bouts (times/day) } \\
\hline Pre & $1.09 \pm 0.97$ & $0.87 \pm 1.37$ & $0.94 \pm 0.89$ & \multirow[t]{2}{*}{0.218} & \multirow[t]{2}{*}{0.654} & \multirow[t]{2}{*}{0.347} \\
\hline Post & $1.22 \pm 1.24$ & $0.81 \pm 1.31$ & $1.20 \pm 1.00$ & & & \\
\hline \multicolumn{7}{|c|}{ Total time of MVPA in bouts (min/day) } \\
\hline Pre & $19.22 \pm 18.00$ & $14.47 \pm 26.26$ & $14.74 \pm 15.46$ & \multirow[t]{2}{*}{0.728} & \multirow[t]{2}{*}{0.584} & 0.476 \\
\hline Post & $20.04 \pm 22.40$ & $12.38 \pm 20.02$ & $17.78 \pm 15.80$ & & & \\
\hline Daily as & & & & & & \\
\hline Pre & $14.57 \pm 4.50$ & $16.84 \pm 3.81$ & $14.86 \pm 5.40$ & 0.403 & 0.268 & 0.728 \\
\hline Post & $14.40 \pm 4.15$ & $16.27 \pm 3.64$ & $14.85 \pm 4.38$ & & & \\
\hline
\end{tabular}

Note: $\mathrm{PG}$ = periodized group; $\mathrm{NPG}=$ non-periodized group; $\mathrm{CG}=$ control group; $\mathrm{SB}=$ sedentary behavior; LPA = light physical activity; $\mathrm{MVPA}$ $=$ moderate to vigorous physical activity; $\Delta \%=$ percentage difference between pre- and post-intervention moments; time $=$ significant difference between pre- and post-intervention moments for variables $\mathrm{PA}, \mathrm{SB}$, bouts and breaks $-\mathrm{p}>0.05$; group = significant difference between groups for variables $\mathrm{PA}, \mathrm{SB}$, bouts and breaks $-\mathrm{p}>0.05 ; \mathrm{TxG}=$ interaction between group and time for variables $\mathrm{PA}, \mathrm{SB}$, bouts and breaks $-\mathrm{p}>0.05$

ual PA levels based on the combination of modalities still present inconclusive data ${ }^{23}$. A study ${ }^{13}$ involving middle-aged men with overweight who had not been previously trained found an increase in total energy expenditure and a tendency of increase in energy expenditure deriving from non-prescribed physical activity. It was also found differences, for these variables, between the training and control groups after 18 weeks of aerobic training. In the present study, independently of the training progression, when we compared two training models, our findings did not show differences between groups in PA and SB levels of adults with obesity. It is important to highlight that, in our study, we assessed
MVPA in bouts, which would be a more accurate indicator of structured PA in terms of duration and intensity, and also total MVPA and LPA, which count any body movement above basal levels, but none of these variables changed with the evaluated training programs.

A possible explanation for these findings is the occurrence of a compensatory change, which can happen after an individual starts a supervised physical training program. Because they think that their involvement in the program is sufficient, they compensate this behavior by reducing global PA levels in other moments of the day and increase $\mathrm{SB}^{14}$. In fact, compensatory change is a recurring factor that directly influences the 
results of programs targeted at weight loss $23-25$. Some training-related variables can act as predictors of such compensatory change, like sex, age, duration of the session, and intervention period ${ }^{14}$.

Regarding sex and age, the increase in habitual PA levels seems to be mitigated in older women, while in younger men and women there is an increase in total daily energy expenditure as a result of physical training $^{14}$. In the present study, the sample was mostly composed of young women and, even so, we did not find an increase in global PA levels. As for the duration of the training session, it seems to be inversely proportional to PA levels, indicating that a longer duration is associated with lower habitual PA levels. However, longer interventions have been more efficient in increasing PA lev$\mathrm{els}^{14}$. Training volume seems to be a strong determinant of PA levels in individuals engaged in physical training programs. Santos et al. ${ }^{26}$ analyzed the effects of one single aerobic session in high and moderate intensities with low training volume in inactive obese adults and no significant changes were identified in PA levels and SB after seven days. In our study, the physical training that was applied was of high volume with moderate intensity, with progression from light to vigorous in one of the groups, but changes in PA levels and reductions in SB time were not observed. Therefore, we suggest that the participants may have stopped practicing exercises immediately after the end of the intervention and did not attempt to be more physically active in a general way, which shows the need to raise this population's awareness in relation to the importance of continuing the training and replacing SB time with engagement in global PAs in order to gain benefits related to the treatment of obesity and to a general improvement in health.

Thus, to achieve greater benefits with the practice of exercises, it is necessary to reduce $\mathrm{SB}$ time in parallel to the training program. Changes in SB time can be achieved with measures that aim to increase PA or to reduce $\mathrm{SB}$, with goals and strategies directed at changes in the home and work environments ${ }^{27}$, like reductions in sitting time and increases in standing or walking time ${ }^{28}$. In addition, motivational aspects are factors that can also favor changes in daily PA. Kerrigan et al. ${ }^{29}$ investigated the effects of a weight loss program based on behavioral education and found that the program significantly reduced total and prolonged $\mathrm{SB}$ and increased MVPA levels. In our study, the presupposition was that a structured and supervised physical training program could change PA levels and reduce SB time, even without goals and strategies targeted at behavior change, but the findings suggest that, to achieve this objective, goals and strategies can be fundamental.

The study had some limitations that cannot be dismissed in the interpretation of the results. Firstly, the adherence of the PG and NPG participants was considered low, represented by an attendance of slightly over $50 \%$ of the training sessions. However, it is not possible to consider this a factor responsible for mitigating the effects of the intervention on the PA levels and SB time that were expected as a result of the training. Another limitation of the study is related to the fact that the number of randomized participants was lower than the number established by the sample size calculation, indicating that the results of the present study might have been influenced by lack of statistical power. It is important to mention that a total of 515 subjects were interested in participating in the study and were submitted to an initial screening. Nevertheless, due to the eligibility criteria and to the fact that some individuals decided not to participate in the study or did not have time to undergo the assessments and/or the intervention, only 83 were considered eligible for the study (Figure 2). Of these, 12 decided not to participate before randomization and two were not available to be assessed. Due to logistics and viability issues, it was not possible to recruit more subjects to be randomized in the same occasion, nor to recruit other subjects afterwards, which resulted in only 69 randomized subjects.

In addition, the participants'low adherence to training and the reduced sample size prevent data extrapolation, indicating low external validity and implying that the study's internal validity should be interpreted with caution. Anyway, this result shows that future research and interventions targeted at this public must be structured in such a way as to implement strategies that increase adherence to exercise programs. In light of this scenario, we recommend that future studies having the variable MVPA as primary outcome should have a minimum sample size of 37 participants per group. Considering the lack of studies with similar designs, involving combined physical training and focusing on improving $\mathrm{PA}$ or $\mathrm{SB}$, this recommendation is based on calculation of minimum sample size with level of significance of $5 \%$, statistical power of $80 \%$ and a conservative effect size (low effect), according to a study that reviewed physical activity promotion interventions ${ }^{30}$.

Moreover, accelerometers provide a limited assessment of activities with overload, hindering the investi- 
gation of the participants'behavior concerning the performance of these activities. Another limitation of the study was precisely the non-inclusion of motivational strategies aiming at changes in participants' $\mathrm{PA}$ and $\mathrm{SB}$ levels. However, it is important to highlight that this study was a secondary analysis of a randomized clinical trial and the analysis of the participants' behavior in relation to $\mathrm{PA}$ was not its main objective.

On the other hand, some strong points deserve to be mentioned here. PA and SB levels were assessed by accelerometry, an objective and highly accurate measure to evaluate habitual and structured activities. In addition, investigating the effects of combined and supervised physical training on this population's PA and SB levels is greatly important, as changing this public's lifestyle is essential to improve health-related outcomes $^{3}$ and the effects of periodization on combined training have not been sufficiently examined.

A supervised program of combined physical training was not able to promote changes in the PA levels and SB time of adults with obesity. Our study presented results that help to understand whether the manipulation of training loads can be determinant in changing this population's PA levels and SB time. We suggest that future studies involving supervised physical training programs targeted at this population should include behavior change strategies that can contribute to lifestyle modifications in a global way. Furthermore, interventions targeted at this public must include measures that favor adherence to training, thus resulting in lower dropout rates.

\section{Conflicts of interest}

The authors declare no conflict of interest.

\section{Authors' contribution}

Barcelos GT participated in the initial conception of the study, data collection, bibliographic search, and in the writing of the manuscript. Del Duca GF participated in the initial conception of the study and in the critical review of the text. Medeiros PRO participated in the initial conception of the study, data collection, bibliographic search, and in the writing of the manuscript. Silva ICM participated in the critical review of the text. Gerage AM participated in the initial conception of the study, data analysis, and in the critical review of the text.

\section{Acknowledgements}

The authors would like to thank all the individuals involved in the study and the research participants.

\section{References}

1. World Health Organization. Obesity and overweight. Disponível em: https://www.who.int/news-room/factsheets/detail/obesity-and-overweight.

2. Goran MI. Energy metabolism and obesity. Med Clin North Am. 2000;84(2):347-62.

3. Yumuk V, Tsigos C, Fried M, Schindler K, Busetto L, Micic D, et al. European Guidelines for Obesity Management in Adults. Obes Facts. 2015;8(6):402-24.

4. Schwingshackl L, Dias S, Strasser B, Hoffmann G. Impact of different training modalities on anthropometric and metabolic characteristics in overweight/obese subjects: a systematic review and network meta-analysis. PLoS ONE. 2013;8(12):e82853.

5. Marzolini S, Oh PI, Brooks D. Effect of combined aerobic and resistance training versus aerobic training alone in individuals with coronary artery disease: a meta-analysis. Eur J Prev Cardiol. 2012;19(1):81-94.

6. Fleck SJ, Kraemer WJ. Fundamentos do treinamento de força muscular. 2o ed. Porto Alegre - RS: Artmed; 1999.

7. Biswas A, Oh PI, Faulkner GE, Bajaj RR, Silver MA, Mitchell MS, et al. Sedentary time and its association with risk for disease incidence, mortality, and hospitalization in adults: a systematic review and meta-analysis. Ann Intern Med. 2015;162(2):123.

8. Wilmot EG, Edwardson CL, Achana FA, Davies MJ, Gorely T, Gray LJ, et al. Sedentary time in adults and the association with diabetes, cardiovascular disease and death: systematic review and meta-analysis. Diabetologia. 2012;55(11):2895-905.

9. Phillips CM, Dillon CB, Perry IJ. Does replacing sedentary behaviour with light or moderate to vigorous physical activity modulate inflammatory status in adults? Int J Behav Nutr Phys Act. 2017;14(1).

10. Yates T, Henson J, Edwardson C, Dunstan D, Bodicoat $\mathrm{DH}$, Khunti K, et al. Objectively measured sedentary time and associations with insulin sensitivity: Importance of reallocating sedentary time to physical activity. Prev Med. 2015;76:79-83.

11. Grgic J, Mikulic P, Podnar H, Pedisic Z. Effects of linear and daily undulating periodized resistance training programs on measures of muscle hypertrophy: a systematic review and meta-analysis. PeerJ. 2017;5:e3695.

12. Levine JA, Lanningham-Foster LM, McCrady SK, Krizan AC, Olson LR, Kane PH, et al. Interindividual variation in posture allocation: possible role in human obesity. science. 2005;307:4.

13. Turner JE, Markovitch D, Betts JA, Thompson D. Nonprescribed physical activity energy expenditure is maintained with structured exercise and implicates a compensatory increase in energy intake. Am J Clin Nutr. 2010;92:1009-16.

14. Fedewa MV, Hathaway ED, Williams TD, Schmidt MD. Effect of exercise training on non-exercise physical activity: a systematic review and meta-analysis of randomized controlled trials. Sports Med. 2017;47(6):1171-82.

15. Streb AR, Silva RP, Leonel LS, Tozetto WR, Gerage AM, Benedet J, et al. Comparison of linear periodized and nonperiodized combined training in health markers and physical fitness of adults with obesity: Clinical trial protocol. Contemp Clin Trials Commun. 2019;15:100358. 
16. Jones AM, Doust JH. A $1 \%$ treadmill grade most accurately reflects the energetic cost of outdoor running. J Sports Sci. 1996;14(4):321-7.

17. Freedson PS, Melanson E, Sirard J. Calibration of the Computer Science and Applications, Inc. accelerometer. Med Sci Sports Exerc. 1998;30(5):777-81.

18. Sasaki JE, John D, Freedson PS. Validation and comparison of ActiGraph activity monitors. J Sci Med Sport. 2011;14(5):411-6.

19. Sasaki J, Coutinho A, Santos C, Bertuol C, Minatto G, Berria J, et al. Orientações para utilização de acelerômetros no Brasil. Rev Bras Ativ Fís Saúde. 2017;22(2):110-26.

20. Enders C. Applied missing data analysis. 1o ed. New York; 2010. 377 p.

21. Almeida FA, You W, Harden SM, Blackman KCA, Davy BM, Glasgow RE, et al. Effectiveness of a worksite-based weight loss randomized controlled trial: The worksite study: Worksite-Based Weight Loss Randomized Controlled Trial. Obesity. 2015;23(4):737-45.

22. Gerage AM, Benedetti TRB, Ritti-Dias RM, Santos ACO, Souza BCC, Almeida FA. Effectiveness of a behavior change program on physical activity and eating habits in patients with hypertension: a randomized controlled trial. J Phys Act Health. 2017;14(12):943-52.

23. Drenowatz C. Reciprocal compensation to changes in dietary intake and energy expenditure within the concept of energy balance. Adv Nutr. 2015;6(5):592-9.

24. Manthou E, Gill JMR, Wright A, Malkova D. Behavioural compensatory adjustments to exercise training in overweight women: Med Sci Sports Exerc. 2010;42(6):1121-28.
25. Dhurandhar EJ, Kaiser KA, Dawson JA, Alcorn AS, Keating $\mathrm{KD}$, Allison DB. Predicting adult weight change in the real world: a systematic review and meta-analysis accounting for compensatory changes in energy intake or expenditure. Int J Obes. 2015;39(8):1181-7.

26. Santos VOA, Browne RAV, Souza DC, Matos VAF, Farias-Junior LF, Farias-Júnior JC, et al. Effects of highintensity interval and moderate-intensity continuous exercise on physical activity and sedentary behavior levels in inactive obese males: a cross- over trial. J Sports Sci Med. 2019;18(3):390-98.

27. Keadle SK, Conroy DE, Buman MP, Dunstan DW, Matthews CE. Targeting reductions in sitting time to increase physical activity and improve health. Med Sci Sports Exerc. 2017;49(8):1572-82.

28. Aunger JA, Doody P, Greig CA. Interventions targeting sedentary behavior in non-working older adults: a systematic review. Maturitas. 2018;116:89-99.

29. Kerrigan SG, Call C, Schaumberg K, Forman E, Butryn ML. Associations between change in sedentary behavior and outcome in standard behavioral weight loss treatment. Transl Behav Med. 2018;8(2):299-304.

30. Sanchez A, Bully P, Martinez C, Grandes G. Effectiveness of physical activity promotion interventions in primary care: a review of reviews. Prev Med. 2015;76: S56-S67.

Received: 21/03/2020

Approved: $15 / 10 / 2020$

\section{Quote this article as: \\ Barcelos GT, Del Duca GF, Medeiros PRO, Crochemore-Silva I, Silva AMG. Effect of physical training periodization on physical activity level in adults with obesity. Rev Bras Ativ Fís Saúde. 2020;25:e0161. DOI: 10.12820/rbafs.25e0161}

